\title{
Static- and Dynamical-Phase Transition in One-Dimensional Reaction-Diffusion Systems with Boundaries
}

\author{
Mohammad Khorrami $^{a}$ and Amir Aghamohammadi ${ }^{b}$ \\ ${ }^{a}$ Institute for Advanced Studies in Basic Sciences, P. O. Box 159, Zanjan 45195, Iran \\ ${ }^{b}$ Department of Physics, Alzahra University, Tehran 19834, Iran
}

Received on 18 March, 2003

\begin{abstract}
A general system of particles (of one or several species) on a one dimensional lattice with boundaries is considered. Two general behaviors of such systems are investigated. The stationary behavior of the system, and the dominant way of the relaxation of the system toward its stationary system. Bases on the first behavior, static phase transitions (discontinuous changes in the stationary profiles of the system) are studied. Based on the second behavior, dynamical phase transitions (discontinuous changes in the relaxation-times of the system) are studied. The investigation is specialized on systems in which the evolution equation of one-point functions are closed (the autonomous systems).
\end{abstract}

\section{Introduction}

The study of the reaction-diffusion systems has been an attractive area. A reaction-diffusion system consists of a collection of particles (of one or several species) moving and interacting with each other with specific probabilities (or rates in the case of continuous time variable). In the so called exclusion processes, any site of the lattice the particles move on, is either vacant or occupied by one particle. The aim of studying such systems, is of course to calculate the time evolution of such systems. But to find the complete time evolution of a reaction-diffusion system, is generally a very difficult (if not impossible) task.

Reaction-diffusion system have been studied using various methods: analytical techniques, approximation methods, and simulation. The success of the approximation methods, may be different in different dimensions, as for example the mean field techniques, working good for high dimensions, generally do not give correct results for low dimensional systems. A large fraction of analytical studies, belong to low-dimensional (specially one-dimensional) systems, as solving low-dimensional systems should in principle be easier [1-13].

Various classes of reaction-diffusion systems are called exactly-solvable, in different senses. In [14] and [15], integrability means that the $N$-particle conditional probabilities' S-matrix is factorized into a product of 2-particle Smatrices. This is related to the fact that for systems solvable in this sense, there are a large number of conserved quantities. In [16-25], solvability means closedness of the evolution equation of the empty intervals (or their generalization).

Consider a reaction-diffusion system (on a lattice) with open boundaries. By open boundaries, it is meant that in addition to the reactions in the bulk of the lattice, particles at the boundaries do react with some external source. A question is to find the possible phase transitions of the system. By phase transition, it is meant a discontinuity in some be- havior of the system with respect to its parameters. Such discontinuities, may arise in two general categories: in the stationary (large time) profiles of the system, and in the time constants determining the evolution of the system. In the first case, static phase transitions are dealt with; in the second case, dynamical phase transitions. As mentioned before, the task of finding the complete evolution of a general reaction-diffusion system is very difficult (if not impossible). So our studies are limited to a certain class of reactiondiffusion systems, and in them to certain properties. To be specific, we deal with one-dimensional systems for which the evolution equation of one-point functions (probabilities of finding a particle of a certain kind on a certain point) is closed (autonomous systems). For these systems, we only consider the final (stationary) profile of the one-point function, and the relaxation-time (the most significant one) towards the stationary profile.

In [26], a ten-parameter family of one-species reactiondiffusion processes with nearest-neighbor interaction was introduced, for which the evolution equation of $n$-point functions contains only $n$ - or less- point functions. The average particle-number in each site has been obtained exactly for these models. In [27, 28], this has been generalized to multi-species systems and more-than-two-site interactions. In [29-32], the phase structure of some classes of singleor multiple-species reaction-diffusion systems have been investigated. These investigations were based on the one-point functions of the systems.

The scheme of the present article is as follows. In section II, the conditions for the most general multi-species reaction-diffusion models with nearest-neighbor interactions, to be solvable, or autonomous, are obtained. In section III, autonomous single-species reaction-diffusion models with boundaries have been investigated. It is shown that changing the bulk rates may lead to a static phase transition, while changing the bulk or boundary rates may lead to a dynamical phase transition. In section IV, as an example, 
an asymmetric generalization of the Glauber model at zero temperature is introduced. This system exhibits both static and dynamical phase transitions. In section $\mathrm{V}$, as an example of systems with more than two-site interactions, Glauber model at finite temperature has been considered. With specific boundary interactions, the Glauber model with open boundaries is also autonomous. It is shown that although this system does not show a static phase transition, it does exhibit a dynamical phase transition. In the last section, as an example of multi-species reaction-diffusion systems, an asymmetric generalization of the zero temperature Potts model is introduced. This system exhibits both a static and a dynamical phase transition.

\section{Autonomous reaction-diffusion systems with nearest-neighbor in- teractions}

In [27], multi-species reaction diffusion systems with nearest-neighbor interactions on a one-dimensional lattice are studied, and criteria are obtained that such systems be autonomous, meaning that the evolution equation for the one-point functions be closed. The summary of the work follows.

Let the Hamiltonian of the system be

$$
\mathcal{H}=\sum_{i=1}^{L-1} H_{i, i+1},
$$

where

$$
H_{i, i+1}:=\underbrace{1 \otimes \cdots \otimes 1}_{i-1} \otimes H \otimes \underbrace{1 \otimes \cdots \otimes 1}_{L-i-1} .
$$

1 is the $(p+1) \times(p+1)$ unit matrix, and $H$ is a $(p+1)^{2} \times(p+1)^{2}$ matrix (for a $p$ species system), the nondiagonal elements of which are nonnegative. $H$ also satisfies

$$
\text { s } H=0 \text {, }
$$

where $\mathbf{s}$ is a covector with all of its components equal to 1 :

$$
s_{\alpha}=1
$$

The number operator of a particle of type $A_{\alpha}$ in the site $i$ is denoted by $n_{i}^{\alpha}$, where $A_{p+1}$ can be the vacancy. These number operators are of the form

$$
n_{i}^{\alpha}:=\underbrace{1 \otimes \cdots \otimes 1}_{i-1} \otimes n^{\alpha} \otimes \underbrace{1 \otimes \cdots \otimes 1}_{L-i}
$$

where $n^{\alpha}$ is a diagonal $(p+1) \times(p+1)$ matrix, the only nonzero element of which is the element $\alpha$ of the diagonal. It is clear that the vector $\mathbf{n}$ whose components are $n^{\alpha}$, satisfies

$$
\mathbf{s} \mathbf{n}=1, \quad \text { or } \quad s_{\alpha} n^{\alpha}=1 .
$$

The Hamiltonian $\mathcal{H}$ is the generator of time translation, by which it is meant that

$$
\frac{\mathrm{d}}{\mathrm{d} t} \mathbf{P}(t)=\mathcal{H} \mathbf{P}(t)
$$

where $\mathbf{P}$ is a the vector in the $(p+1)^{L}$ dimensional space, the component $P^{\alpha_{1} \cdots \alpha_{L}}$ of which is the probability of finding a particle of type $A_{\alpha_{1}}$ in site 1 , etc. Clearly, the components of this vector are nonnegative and satisfy

$$
\mathbf{S P}=1
$$

where

$$
\mathbf{S}:=\underbrace{\mathbf{s} \otimes \cdots \otimes \mathbf{s}}_{L} .
$$

The fact that $\mathbf{P}$ should have these properties for all times, means that the nondiagonal elements of $\mathcal{H}$ should be nonnegative and that the action of $\mathcal{H}$ (from right) on $\mathbf{S}$ should be zero. But the nonnegativity of the nondiagonal elements of $H$, and the condition (3), are sufficient for these two conditions on $\mathcal{H}$ to be satisfied.

The expectation value of any operator $\mathcal{O}$ is

$$
\langle\mathcal{O}\rangle=\mathbf{S} \mathcal{O} \mathbf{P}
$$

This is also true for $n_{i}^{\alpha}$, from which it is seen that

$$
\frac{\mathrm{d}}{\mathrm{d} t}\left\langle n_{i}^{\alpha}\right\rangle=\mathbf{S} n_{i}^{\alpha} \mathcal{H} \mathbf{P}
$$

Now the question is, under what conditions the right-hand side of (11) can be written in terms of the one-point functions $n_{j}^{\beta}$, s?

To answer this, one notes that $n_{i}^{\alpha}$ commutes with all of terms in $\mathcal{H}$, except possibly with $H_{i-1, i}$ and $H_{i, i+1}$. Using

$$
\mathbf{s} \otimes \mathbf{s}(\mathbf{a} \mathbf{n}) \otimes(\mathbf{b} \mathbf{n}) H=(\mathbf{a} \otimes \mathbf{b} H)_{\alpha \beta} \mathbf{s} \otimes \mathbf{s} \mathbf{n}^{\alpha} \otimes \mathbf{n}^{\beta},
$$

which is true for any two covectors $\mathbf{a}$ and $\mathbf{b}$, and using (6), it is seen that

$$
\frac{\mathrm{d}}{\mathrm{d} t}\left\langle n_{i}^{\alpha}\right\rangle=s_{\gamma} H_{\mu \nu}^{\gamma \alpha}\left\langle n_{i-1}^{\mu} n_{i}^{\nu}\right\rangle+s_{\gamma} H_{\mu \nu}^{\alpha \gamma}\left\langle n_{i}^{\mu} n_{i+1}^{\nu}\right\rangle .
$$

The right hand side is expressed in terms of two-point functions not one-point functions. However, from (6) it is seen that the two-point functions are not independent. In order that an expression $B_{\mu \nu} n_{i}^{\mu} n_{j}^{\nu}$ be expressible in terms of the first power of number operators, it is necessary and sufficient that

$$
B_{\mu \nu}={ }_{1} B_{\mu} s_{\nu}+s_{\mu 2} B_{\nu} .
$$

So the necessary and sufficient condition for the system to be autonomous, is that

$$
{ }^{i} H^{\alpha}{ }_{\mu \nu}={ }_{1}^{i} H^{\alpha}{ }_{\mu} s_{\nu}+s_{\mu}{ }_{2}^{i} H^{\alpha}{ }_{\nu}
$$

where

$$
\begin{aligned}
{ }^{1} H^{\alpha}{ }_{\mu \nu} & :=s_{\gamma} H^{\alpha \gamma}{ }_{\mu \nu}, \\
{ }^{2} H^{\alpha}{ }_{\mu \nu} & :=s_{\gamma} H^{\gamma \alpha}{ }_{\mu \nu} .
\end{aligned}
$$

Note that even if (15) is satisfied, ${ }_{j}^{i} H$ 's are not uniquely determined through which. One can change them like

$$
{ }_{j}^{i} H_{\mu}^{\alpha} \rightarrow{ }_{j}^{i} H^{\alpha}{ }_{\mu}+{ }_{j}^{i} w^{\alpha} s_{\mu},
$$


with

$$
\sum_{j}^{i} \mathbf{w}=\mathbf{0}
$$

For the simplest case $p=1$ (single-species systems) (15) consists of two independent constraints, leaving a tenparameter family of autonomous systems out of the twelve- parameter family of the general systems.

The condition (15) for a system to be autonomous, can be easily extended to systems with interaction-ranges longer than the nearest neighbor. This has been done in [28]. The result is that if the interaction is in a block of $k$ neighboring sites, then one should have

where

$$
{ }^{i} H^{\alpha}{ }_{\beta_{1} \cdots \beta_{k}}={ }_{1}^{i} H^{\alpha}{ }_{\beta_{1}} s_{\beta_{2}} \cdots s_{\beta_{k}}+\cdots+s_{\beta_{1}} \cdots s_{\beta_{k-1}}{ }_{k}^{i} H^{\alpha} \beta_{k},
$$

${ }^{i} H^{\alpha_{i}}{ }_{\beta_{1} \cdots \beta_{k}}:=s_{\alpha_{1}} \cdots s_{\alpha_{i-1}} s_{\alpha_{i+1}} \cdots s_{\alpha_{k}} H^{\alpha_{1} \cdots \alpha_{k}}{ }_{\beta_{1} \cdots \beta_{k}}$.

Assuming that (15) holds, one can write the evolution equation for the one-point functions as

$$
\frac{\mathrm{d}}{\mathrm{d} t}\left\langle\mathbf{n}_{i}\right\rangle={ }_{1}^{2} H\left\langle\mathbf{n}_{i-1}\right\rangle+\left({ }_{2}^{2} H+{ }_{1}^{1} H\right)\left\langle\mathbf{n}_{i}\right\rangle+{ }_{2}^{1} H\left\langle\mathbf{n}_{i+1}\right\rangle, \quad 1<i<L .
$$

For $i=1$, at the right-hand only the last two terms remain; for $i=L$, only the first two terms. This is a linear differential-difference equation for the vectors $\langle\mathbf{n}\rangle$.

\section{Autonomous single-species reaction-diffusion systems with boundaries}

For a single-species system, the condition (15) can be rewritten in the more explicit form

$$
\begin{aligned}
& -H^{01}{ }_{11}-H^{00}{ }_{11}+H^{01}{ }_{10}+H^{00}{ }_{10}-H^{11}{ }_{01}-H^{10}{ }_{01}+H^{11}{ }_{00}+H^{10}{ }_{00}=: 0 \\
& -H^{10}{ }_{11}-H^{00}{ }_{11}-H^{11}{ }_{10}-H^{01}{ }_{10}+H^{10}{ }_{01}+H^{00}{ }_{01}+H^{11}{ }_{00}+H^{01}{ }_{00}=: 0 .
\end{aligned}
$$

Here the state $A_{1}$ is an occupied site, while $A_{0}$ is a vacancy. Defining

$$
\begin{aligned}
u & :=H^{10}{ }_{01}+H^{00}{ }_{01} \\
v & :=H^{01}{ }_{10}+H^{00}{ }_{10} \\
\bar{u} & :=H^{10}{ }_{11}+H^{00}{ }_{11} \\
\bar{v} & :=H^{01}{ }_{11}+H^{00}{ }_{11} \\
w & :=H^{11}{ }_{00}+H^{10}{ }_{00} \\
s & :=H^{11}{ }_{00}+H^{01}{ }_{00} \\
\bar{w} & :=H^{11}{ }_{01}+H^{10}{ }_{01} \\
\bar{s} & :=H^{11}{ }_{10}+H^{01}{ }_{10},
\end{aligned}
$$

one can write (22) as

$$
\begin{aligned}
u+s & =\bar{u}+\bar{s} \\
v+w & =\bar{v}+\bar{w} .
\end{aligned}
$$

The evolution equation of $\left\langle\mathbf{n}_{i}\right\rangle$, for $1<i<L$ is of the form (21). For $i=1$ and $i=L$, only half of the terms at the right-hand side of (21) are there. Moreover, if there is injection and extraction of particles at the end sites, terms corresponding to these rates should also be added. Finally, for the single species case, denoting $n_{i}^{1}$ by $n_{i}$, one can write instead of the vector equation (21), a scalar equation for $n_{i}$, as $n_{i}^{1}+n_{i}^{0}=1$. denoting the injection and extraction rates at the first site by $a$ and $a^{\prime}$ respectively, and those at the last site by $b$ and $b^{\prime}$, the evolution equation of the one-point functions can be seen to be

$$
\begin{aligned}
\left\langle\dot{n}_{i}\right\rangle= & -(v+w+u+s)\left\langle n_{i}\right\rangle+(v-\bar{v})\left\langle n_{i+1}\right\rangle+(u-\bar{u})\left\langle n_{i-1}\right\rangle \\
& +w+s, \quad 1<i<L \\
\left\langle\dot{n}_{1}\right\rangle= & -(v+w)\left\langle n_{1}\right\rangle+(v-\bar{v})\left\langle n_{2}\right\rangle+w+a\left(1-\left\langle n_{1}\right\rangle\right)-a^{\prime}\left\langle n_{1}\right\rangle \\
\left\langle\dot{n}_{L}\right\rangle= & -(u+s)\left\langle n_{L}\right\rangle+(u-\bar{u})\left\langle n_{L-1}\right\rangle+s+b\left(1-\left\langle n_{L}\right\rangle\right)-b^{\prime}\left\langle n_{L}\right\rangle,
\end{aligned}
$$




\section{III.1 The static phase transition}

The stationary-state to (25) is

$$
\left\langle n_{j}\right\rangle=C+D_{1} z_{1}^{j}+D_{2} z_{2}^{j},
$$

where $z_{i}$ 's satisfy

$$
-r+p z_{i}+q z_{i}^{-1}=0,
$$

and we take $z_{2}$ to be the root of the above equation with larger absolute value, and the new parameters $p, q$, and $r$ are defined through

$$
\begin{aligned}
p & :=v-\bar{v} \\
q & :=u-\bar{u} \\
r & :=u+s+v+w=u+s+\bar{v}+\bar{w} \\
& =\bar{u}+\bar{s}+v+w=\bar{u}+\bar{s}+\bar{v}+\bar{w},
\end{aligned}
$$

Noting that the rates (nondiagonal elements of $H$ ) are nonnegative, it is seen that

$$
r \geq|p+q|,|p-q| \text {. }
$$

More over, it is seen that if $r=0$, then all the parameters introduced in (23) vanish, and $\left\langle n_{i}\right\rangle$ would be constant (for $1<i<L$ ). So apart from this trivial case, $r$ is positive and in fact can be rescaled to one (by a proper redefinition of time). Hence, as long as only the stationary profile of the one-point function is considered, there are only twoparameters in the system:

$$
x:=\frac{p}{r}, \quad \text { and } \quad y:=\frac{q}{r} .
$$

The physical region corresponding to these parameters is a square the boundaries of which are $|x \pm y|=1$.

It can be seen that inside the physical square, both of the roots of (27) are real, one $\left(z_{1}\right)$ between -1 and 1 , the other $\left(z_{2}\right)$ outside that region. So in the thermodynamic limit $L \rightarrow \infty$

$$
\begin{array}{lrl}
\left\langle n_{j}\right\rangle \approx C+D_{1} z_{1}^{j}, & j \ll L \\
\left\langle n_{j}\right\rangle \approx C+D_{2} z_{2}^{j-L-1}, & L-j \ll L .
\end{array}
$$

$C, D_{i}$ 's, and $z_{i}$ 's are smooth functions of rates, and there is no phase transition.

However, if $|x+y|=1$, then one of the roots of (27) would be $x+y$; that is, the absolute value of one of the roots will be 1 . The other roots would be $y / x$. If $|y / x|<1$, then $z_{1}=1$ and from (31) it is seen that near the end of the lattice, $\left|\left\langle n_{j}\right\rangle-C\right|$ is essentially constant. If $|y / x|>1$, then $z_{2}=1$ and from (31) it is seen that near the beginning of the lattice, $\left|\left\langle n_{j}\right\rangle-C\right|$ is essentially constant. So there is a phase transition at $x=y= \pm 1 / 2$. This static phase transition manifests itself as a change in the slope of the profile of the one-point function, near the end or the beginning of the lattice.

\section{III.2 The dynamical phase transition}

The dynamical phase transition is related to the relaxation time of the system evolving towards its stationary configuration. To find this relaxation time, one writes the homogeneous part of (25) as

$$
\dot{N}_{i}=h_{i}{ }^{j} N_{j}
$$

where $N_{i}$ is $\left\langle n_{i}\right\rangle$ minus its stationary value. Then, one seeks the eigenvalues of $h$. The eigenvalue with the largest real part, determines the relaxation time:

$$
\tau=-\frac{1}{\operatorname{Re}\left(E_{\max }\right)}
$$

The eigenvector equations read

$$
\begin{aligned}
& E X_{j}=-(v+w+u+s) X_{j}+(v-\bar{v}) X_{j+1}+(u-\bar{u}) X_{j-1}, \quad j \neq 1, L \\
& E X_{1}=-\left(v+w+a+a^{\prime}\right) X_{1}+(v-\bar{v}) X_{2}, \\
& E X_{L}=-\left(u+s+b+b^{\prime}\right) X_{L}+(u-\bar{u}) X_{L-1} .
\end{aligned}
$$

A solution for this is

$$
X_{j}=\alpha z_{1}^{j}+\beta z_{2}^{j}
$$

with

$$
E=-(v+w+u+s)+(v-\bar{v}) z_{i}+(u-\bar{u}) z_{i}^{-1},
$$

and

$$
\begin{aligned}
(v-\bar{v})\left(\alpha z_{1}^{2}+\beta z_{2}^{2}\right)-\left(E+a+a^{\prime}+v+w\right)\left(\alpha z_{1}+\beta z_{2}\right) & =0 \\
(u-\bar{u})\left(\alpha z_{1}^{L-1}+\beta z_{2}^{L-1}\right)-\left(E+b+b^{\prime}+u+s\right)\left(\alpha z_{1}^{L}+\beta z_{2}^{L}\right) & =0 .
\end{aligned}
$$

One puts $E$ from (36) in (37), and demands (37) to have nonzero solutions for $\alpha$ and $\beta$. The result is

$$
\begin{aligned}
{\left[(u-\bar{u})+z_{1} \delta a\right]\left[(v-\bar{v}) z_{2}^{L+1}+z_{2}^{L} \delta b\right] } & -\left[(u-\bar{u})+z_{2} \delta a\right] \\
\times & {\left[(v-\bar{v}) z_{1}^{L+1}+z_{1}^{L} \delta b\right]=0, }
\end{aligned}
$$


where

$$
\begin{aligned}
\delta a & :=a+a^{\prime}-(u+s), \\
\delta b & :=b+b^{\prime}-(v+w) .
\end{aligned}
$$

Defining the new variables $Z_{i}$ as

$$
Z_{i}:=z_{i} \sqrt{\left|\frac{v-\bar{v}}{u-\bar{u}}\right|}
$$

it is seen that

$$
\begin{aligned}
E & =-(v+w+u+s)+\sqrt{|(u-\bar{u})(v-\bar{v})|}\left[Z_{i} \operatorname{sgn}(v-\bar{v})+Z_{i}^{-1} \operatorname{sgn}(u-\bar{u})\right] \\
& =-(v+w+u+s)+\operatorname{sgn}(v-\bar{v}) \sqrt{|(u-\bar{u})(v-\bar{v})|}\left(Z_{1}+Z_{2}\right) \\
& =-(v+w+u+s)+\operatorname{sgn}(u-\bar{u}) \sqrt{|(u-\bar{u})(v-\bar{v})|}\left(Z_{1}^{-1}+Z_{2}^{-1}\right) .
\end{aligned}
$$

In the thermodynamic limit $L \rightarrow \infty$, all unimodular complex numbers are solutions for $Z_{i}$. The maximum of the real part of $E$ depends on whether all of the values of $Z_{i}$ are unimodular or not. In the first case, the relaxation time is independent of the injection and extraction rates at the boundaries. This is called the fast phase. In the second case, the relaxation time does depend on the injection and extraction rates at the boundaries. This is called the slow phase. The terms fast and slow come from the fact that for fixed bulk reaction rates, the relaxation time in the fast phase is smaller than that of the slow phase. Now the physical parameter space can be divided to three regions, with different behaviors for the relaxation-time:

$$
\left\{\begin{array}{lll}
a+a^{\prime}<\mathcal{A}, & a+a^{\prime}-\left(b+b^{\prime}\right)<\mathcal{A}-\mathcal{B}, & \text { region I } \\
b+b^{\prime}<\mathcal{B}, & a+a^{\prime}-\left(b+b^{\prime}\right)>\mathcal{A}-\mathcal{B}, & \text { region II } \\
\text { otherwise, } & \text { region III }
\end{array}\right.
$$

where

$$
\begin{aligned}
& \mathcal{A}:=u+s-\sqrt{|(u-\bar{u})(v-\bar{v})|}=\bar{u}+\bar{s}-\sqrt{|(u-\bar{u})(v-\bar{v})|} \\
& \mathcal{B}:=v+w-\sqrt{|(u-\bar{u})(v-\bar{v})|}=\bar{v}+\bar{w}-\sqrt{|(u-\bar{u})(v-\bar{v})|} .
\end{aligned}
$$

The relaxation time $\tau$ is then

$$
\tau= \begin{cases}{\left[v+w+a+a^{\prime}+(u-\bar{u})(v-\bar{v})\left(a+a^{\prime}-u-s\right)^{-1}\right]^{-1},} & \text { region I } \\ {\left[u+s+b+b^{\prime}+(u-\bar{u})(v-\bar{v})\left(b+b^{\prime}-v-w\right)^{-1}\right]^{-1},} & \text { region II } \\ \{v+w+u+s-2 \operatorname{Re}[\sqrt{(u-\bar{u})(v-\bar{v})}]\}^{-1}, & \text { region III }\end{cases}
$$

It is seen that in region I, the relaxation time depends on the injection and extraction rates at the beginning of the lattice; in region II it depends on the injection and extraction rates at the end of the lattice; and in region III it depends on none of the injection or extraction rates.

The details of the calculations can be seen in [30].

\section{An asymmetric generalization of the Glauber model at zero temper- ature, as an example}

The Glauber model [33] is a model for the relaxation of an Ising model towards its equilibrium with a heat bath at tem- perature $T$, which is based on the principle of detailed balance. This model is based on a three-site interaction: a spin is flipped with the rate $\mu:=1-\tanh \beta J$ if the spins of its neighboring sites are the same as itself; it flips with the rate $\lambda:=1+\tanh \beta J$, if the the spins of the neighboring sites are opposite to it: and flips with the rate 1 , if the spins of the neighboring sites are opposite to each other. Here $\beta=\left(k_{\mathrm{B}} T\right)^{-1}$, and time has been rescaled so that one of the rates is 1 .

It has been shown in [34] that at zero temperature, the Glauber dynamics is effectively a two-site interaction, in which two opposite neighboring spins become the same, with the rate 1 (independent of which is up and which is down, and which one flips).

An extension of the Glauber dynamics at zero tempera- 
ture is a system with the following dynamics.

$$
\begin{gathered}
A \emptyset \rightarrow \begin{cases}A A, & \text { with the rate } u \\
\emptyset \emptyset, & \text { with the rate } v\end{cases} \\
\emptyset A \rightarrow \begin{cases}A A, & \text { with the rate } v \\
\emptyset \emptyset, & \text { with the rate } u\end{cases}
\end{gathered}
$$

where $A$ denotes a spin up (or a particle) and $\emptyset$ denotes a spin down (or a vacancy). In the ordinary Glauber model, the system is left-right symmetric and $u=v$. Now consider a system with the dynamics (45), on an $L$-site lattice. Assume also that there are injection and extraction rates at the first and last sites, with the rates $a, a^{\prime}, b$, and $b^{\prime}$ introduced in the previous section. It is easily seen that for this system, of the eight bulk-rate parameters introduced in the previous section, only $u, v, \bar{s}$, and $\bar{w}$ are nonzero, and

$$
\begin{aligned}
\bar{s} & =u, \\
\bar{w} & =v .
\end{aligned}
$$

From this, it is seen that

$$
\begin{aligned}
& x=\frac{v}{u+v}, \\
& y=\frac{u}{u+v} .
\end{aligned}
$$

So $x$ and $y$ are nonnegative and $x+y=1$. This means that there is a static phase transition, which occurs at $u=v$, the point corresponding to the ordinary Glauber model.

For the dynamical phase transition, it is seen that $\mathcal{A}$ and $\mathcal{B}$ introduced in (43), are

$$
\begin{aligned}
& \mathcal{A}:=u-\sqrt{u v}, \\
& \mathcal{B}:=v-\sqrt{u v} .
\end{aligned}
$$

Of $\mathcal{A}$ and $\mathcal{B}$, only one can be positive, and this is when $u \neq v$. So, as the injection and extraction rates are nonnegative, at most two of the regions I, II, and III may exist: for $u>v$, only the regions I and III; for $u<v$, only the regions II and III. The expression for the relaxation time is

$$
\tau= \begin{cases}{\left[v+a+a^{\prime}+u v\left(a+a^{\prime}-u\right)^{-1}\right]^{-1},} & \text { region I } \\ {\left[u+b+b^{\prime}+u v\left(b+b^{\prime}-v\right)^{-1}\right]^{-1},} & \text { region II } \\ (v+u-2 \sqrt{u v})^{-1}, & \text { region III }\end{cases}
$$

As a final note, it is easily seen that changing the dynamics (45) into the following

$$
\begin{gathered}
A \emptyset \rightarrow \begin{cases}\emptyset A, & \text { with the rate } \lambda \\
A A, & \text { with the rate } u-\lambda \\
\emptyset \emptyset, & \text { with the rate } v-\lambda\end{cases} \\
\emptyset A \rightarrow \begin{cases}A \emptyset, & \text { with the rate } \lambda^{\prime} \\
A A, & \text { with the rate } v-\lambda^{\prime} \\
\emptyset \emptyset, & \text { with the rate } u-\lambda^{\prime}\end{cases}
\end{gathered}
$$

which contains diffusion as well, does not change the evolution equation of the one-point function. So the results of this section are valid for this interaction as well. The only difference is that the introduction of diffusion, restricts the parameter space of $u$ and $v$, since the rates should be nonnegative. So $u, v \geq \lambda, \lambda^{\prime}$.

\section{Extension to longer-range interac- tions: dynamical phase transition of kinetic Ising model with bound- aries}

It was pointed out in the previous section, that the Glauber dynamics at nonzero temperatures consists of a three-site interaction. Let us rewrite the reactions:

$$
\begin{aligned}
& \uparrow \uparrow \uparrow \rightarrow \uparrow \downarrow \uparrow \quad \text { and } \downarrow \downarrow \downarrow \rightarrow \downarrow \uparrow \downarrow \quad \text { with the rate } \mu \text {, } \\
& \uparrow \downarrow \uparrow \rightarrow \uparrow \uparrow \uparrow \quad \text { and } \downarrow \uparrow \downarrow \rightarrow \downarrow \downarrow \downarrow \quad \text { with the rate } \lambda \text {, } \\
& \uparrow \uparrow \downarrow \rightleftharpoons \uparrow \downarrow \downarrow \text { and } \downarrow \downarrow \uparrow \rightleftharpoons \downarrow \uparrow \uparrow \quad \text { with the rate } 1 \text {, }
\end{aligned}
$$

where an upward arrow means spin up, a downward arrow means spin down, and $\lambda$ and $\mu$ are defined true

$$
\begin{aligned}
\lambda & :=1+\tanh \beta J, \\
\mu & :=1-\tanh \beta J,
\end{aligned}
$$

as in the previous section. As it is noted in [33], the evolution equation for the expectation value of the spins (each spin is either 1 (upward) or -1 (downward)) is closed. This is of course true for the spins at the bulk of the lattice. At the boundaries, one should introduce some two-site interactions and write separate equations for $\left\langle s_{1}\right\rangle$ and $\left\langle s_{L}\right\rangle$. The new interactions introduced at the boundaries are

$$
\begin{aligned}
& \uparrow \downarrow \rightarrow \downarrow \downarrow \quad \text { with the rate } g_{1}, \\
& \uparrow \uparrow \rightarrow \downarrow \uparrow \quad \text { with the rate } g_{2}, \\
& \downarrow \uparrow \rightarrow \uparrow \uparrow \quad \text { with the rate } g_{3}, \\
& \downarrow \downarrow \rightarrow \uparrow \downarrow \quad \text { with the rate } g_{4},
\end{aligned}
$$

For the spin flip of the first site, and

$$
\begin{array}{ll}
\downarrow \uparrow \rightarrow \downarrow \downarrow & \text { with the rate } h_{1}, \\
\uparrow \uparrow \rightarrow \uparrow \downarrow & \text { with the rate } h_{2}, \\
\uparrow \downarrow \rightarrow \uparrow \uparrow & \text { with the rate } h_{3}, \\
\downarrow \downarrow \rightarrow \downarrow \uparrow & \text { with the rate } h_{4},
\end{array}
$$

For the spin flip of the last site. It can be seen that the evolution equation for the one-point functions is closed, iff

$$
\begin{aligned}
g_{1}+g_{4} & =g_{2}+g_{3}, \\
h_{1}+h_{4} & =h_{2}+h_{3} .
\end{aligned}
$$

Provided this is true, the evolution equation is 


$$
\begin{aligned}
\left\langle\dot{s}_{i}\right\rangle & =-2\left\langle s_{i}\right\rangle+\left(\left\langle s_{i+1}\right\rangle+\left\langle s_{i-1}\right\rangle\right) \tanh (2 \beta J), \quad 1<i<L \\
\left\langle\dot{s}_{1}\right\rangle & =-\left(g_{2}+g_{3}\right)\left\langle s_{1}\right\rangle+\left(g_{1}-g_{2}\right)\left\langle s_{2}\right\rangle+\left(g_{3}-g_{1}\right), \\
\left\langle\dot{s}_{L}\right\rangle & =-\left(h_{2}+h_{3}\right)\left\langle s_{L}\right\rangle+\left(h_{1}-h_{2}\right)\left\langle s_{L-1}\right\rangle+\left(h_{3}-h_{1}\right) .
\end{aligned}
$$

The stationary solution for this is

$$
\left\langle s_{j}\right\rangle=D_{1} z_{1}^{k}+D_{2} z_{2}^{k-L-1}
$$

where

$$
z_{1}=z_{2}^{-1}=\tanh (\beta J) .
$$

$D_{1}$ and $D_{2}$ are easily obtained at the thermodynamic limit $L \rightarrow \infty$; and it is seen that they are both smooth functions of the rates. So there is no static phase transition.

For the dynamical phase transition, similar to the method used in the previous sections, one writes the eigenvalue problem for the homogeneous equation. That equation reads

$$
\begin{aligned}
& E X_{j}=-2 X_{j}+\tanh (2 \beta J)\left(X_{j+1}+X_{j-1}\right), \quad j \neq 1, L, \\
& E X_{1}=-\left(g_{2}+g_{3}\right) X_{1}+\left(g_{1}-g_{2}\right) X_{2} \\
& E X_{L}=-\left(h_{2}+h_{3}\right) X_{L}+\left(h_{1}-h_{2}\right) X_{L-1} .
\end{aligned}
$$

The solution to this is an expression like (35), with

$$
E=-2+\tanh (2 \beta J)\left(z_{i}+z_{i}^{-1}\right) .
$$

and

$$
\begin{array}{r}
z_{i}^{1-L}\left[2-g_{2}-g_{3}+z\left(g_{1}-g_{2}-\tanh (2 \beta J)\right)-z_{i}^{-1} \tanh (2 \beta J)\right] \\
\times\left[2-h_{2}-h_{3}+z_{i}\left(h_{1}-h_{2}-\tanh (2 \beta J)\right)-z_{i}^{-1} \tanh (2 \beta J)\right] \\
-z_{i}^{L-1}\left[2-g_{2}-g_{3}+z^{-1}\left(g_{1}-g_{2}-\tanh (2 \beta J)\right)-z_{i} \tanh (2 \beta J)\right] \\
\times\left[2-h_{2}-h_{3}+z_{i}^{-1}\left(h_{1}-h_{2}-\tanh (2 \beta J)\right)-z_{i} \tanh (2 \beta J)\right]=0 .
\end{array}
$$

Again in the thermodynamic limit $L \rightarrow \infty$, if all of the solutions to the above equation are unimodular, the relaxation time would be

$$
\tau=[-2+2 \tanh (2 \beta J)]^{-1},
$$

which is independent of the interaction rates at the boundaries. If there are some solutions that are nonunimodolar, and among them there is a solution with $\operatorname{Re}\left(z_{i}+z_{i}^{-1}\right)>2$ (for the ferromagnetic case $J>0$ ), then the relaxation time becomes larger than (62). This is the slow phase. The criterion for this, is that one of the roots of (61) passes the boundary

$$
y= \pm(x-1) \sqrt{\frac{x}{2-x}},
$$

where $x$ and $y$ are the real and imaginary parts of that root, respectively.

In the thermodynamic limit $L \rightarrow \infty$, the equation (61) for roots with modulus greater than one simplifies into

$$
\begin{aligned}
& \left\{2-g_{2}-g_{3}+z^{-1}\left[g_{1}-g_{2}-\tanh (2 \beta J)\right]-z \tanh (2 \beta J)\right\} \\
\times & \left\{2-h_{2}-h_{3}+z^{-1}\left[h_{1}-h_{2}-\tanh (2 \beta J)\right]-z \tanh (2 \beta J)\right\}=0 .
\end{aligned}
$$

So the criterion for the transition to occur, is that a complex number $z=x+i y$, satisfies (63) and (64). The relation between rates, for this to occur, can be seen to be

$$
\begin{aligned}
& 2[1-\tanh (2 \beta J)]-g_{2}-g_{4}=0, \quad \text { or, } \\
& {\left[4 \tanh (2 \beta J)-2+g_{2}+g_{3}\right]\left[g_{1}-g_{2}-\tanh (2 \beta J)\right]+\left(2-g_{2}-g_{3}\right) \tanh (2 \beta J)=0 .}
\end{aligned}
$$


This is for the case when the first factor in (64) vanishes. A similar criterion come from the vanishing of the second factor, with the roles of $g_{i}$ 's and $h_{i}$ 's interchanged.

The detailed calculations can be found in [32].

\section{Extension to multi-species sys- tems: phase transition in an asym- metric generalization of the zero- temperature Potts model}

A simple extension of the asymmetric generalization of the Glauber model at zero temperature, introduced in section 4 , is an asymmetric generalization of the zero-temperature Potts model. In this system, any site can have $p+1$ states (rather than the two states of the Glauber model). The bulk reactions are written as

$$
A_{\alpha} A_{\beta} \rightarrow\left\{\begin{array}{ll}
A_{\alpha} A_{\alpha}, & \text { with the rate } u \\
A_{\beta} A_{\beta}, & \text { with the rate } v
\end{array} .\right.
$$

Compare this with (45). One adds to this, reaction rates at the boundaries:

$$
\begin{aligned}
& A_{\beta} \rightarrow A_{\alpha} \quad \text { with the rate } \Lambda_{\beta}^{\alpha} \text { at the first site, } \\
& A_{\beta} \rightarrow A_{\alpha} \quad \text { with the rate } \Gamma^{\alpha}{ }_{\beta} \text { at the last site. }
\end{aligned}
$$

For $\alpha \neq \beta, \Lambda^{\alpha}{ }_{\beta}$ and $\Gamma^{\alpha}{ }_{\beta}$ are rates, and should be nonnegative. The diagonal elements of $\Lambda$ and $\Gamma$ are chosen so that

$$
\mathbf{s} \Lambda=\mathbf{s} \Gamma=0 .
$$

Using these, the evolution equation for the one-point functions is written as

$$
\begin{aligned}
\left\langle\dot{\mathbf{n}}_{j}\right\rangle & =-(u+v)\left\langle\mathbf{n}_{j}\right\rangle+u\left\langle\mathbf{n}_{j-1}\right\rangle+v\left\langle\mathbf{n}_{j+1}\right\rangle, \quad j \neq 1, L \\
\left\langle\dot{\mathbf{n}}_{1}\right\rangle & =\Lambda\left\langle\mathbf{n}_{1}\right\rangle-v\left\langle\mathbf{n}_{1}\right\rangle+v\left\langle\mathbf{n}_{2}\right\rangle, \\
\left\langle\dot{\mathbf{n}}_{L}\right\rangle & =\Gamma\left\langle\mathbf{n}_{L}\right\rangle-u\left\langle\mathbf{n}_{L}\right\rangle+u\left\langle\mathbf{n}_{L-1}\right\rangle .
\end{aligned}
$$

\section{VI.1 The static phase transition}

The stationary solution to (68) can be written as

$$
\left\langle\mathbf{n}_{j}\right\rangle=\mathbf{B}+\mathbf{C}^{\prime}\left(\frac{u}{v}\right)^{j-1},
$$

or

$$
\left\langle\mathbf{n}_{j}\right\rangle=\mathbf{B}+\mathbf{C}^{\prime \prime}\left(\frac{u}{v}\right)^{j-L},
$$

where

$$
\begin{aligned}
\mathbf{s} \mathbf{B} & =1, \\
\mathbf{s} \mathbf{C}^{\prime}=\mathbf{s} \mathbf{C}^{\prime \prime} & =0,
\end{aligned}
$$

and $\mathbf{B}$ and $\mathbf{C}^{\prime}$ (or $\mathbf{C}^{\prime \prime}$ ) must also satisfy conditions coming from the evolution equation (68) at the boundaries. For $u<v$, it is better to work with (69). Then it is seen that in the thermodynamic limit $L \rightarrow \infty$, one has

$$
\begin{aligned}
\Gamma \mathbf{B} & =0, \\
(\Lambda-v+u) \mathbf{C}^{\prime} & =-\Lambda \mathbf{B} .
\end{aligned}
$$

The first equation has at least one non-zero solution for $\mathbf{B}$, as $\gamma$ does have a left eigenvector $\mathbf{s}$, with the eigenvalue zero. Depending on the degeneracy of this zero eigenvalue, there are one or several solutions for $\mathbf{B}$. One should then put $\mathbf{B}$ in the second equation to find $\mathbf{C}^{\prime}$. The conditions on $\Lambda$, ensure that the real part of the eigenvalues of $\Lambda$ are nonpositive, so $v-u$ cannot be an eigenvalue of $\Lambda$. Hence, the second equation has one and only one solution for $\mathbf{C}^{\prime}$ (corresponding to each solution for $\mathbf{B}$ ).

For $u>v$, one uses (70) and (in the thermodynamic limit) arrives at

$$
\begin{aligned}
\Lambda \mathbf{B} & =0, \\
(\Gamma-u+v) \mathbf{C}^{\prime \prime} & =-\Gamma \mathbf{B} .
\end{aligned}
$$

Similar arguments hold for the solution of this equation, with the roles of $\Lambda$ and $\Gamma, u$ and $v$, and $\mathbf{C}^{\prime}$ and $\mathbf{C}^{\prime \prime}$ interchanged. Now apart from the question of the uniqueness of the stationary profile (which depends on the degeneracy of the matrices $\Gamma$ or $\Lambda$ ) one can see that if $u<v$, the profile is flat near the last site, while for $u>v$ it is flat near the first sites. This is the static phase transition, similar to what observed for the asymmetric generalization of the zerotemperature Glauber model.

\section{VI.2 The dynamical phase transition} (69) is

The eigenvalue equation for the homogeneous part of

$$
\begin{aligned}
& E \mathbf{X}_{j}=-(u+v) \mathbf{X}_{j}+u \mathbf{X}_{k-1}+v \mathbf{X}_{k+1}, \quad j \neq 1, L \\
& E \mathbf{X}_{1}=\Lambda \mathbf{X}_{1}-v \mathbf{X}_{1}+v \mathbf{X}_{2}, \\
& E \mathbf{X}_{L}=\Gamma \mathbf{X}_{L}-u \mathbf{X}_{L}+u \mathbf{X}_{L-1}
\end{aligned}
$$

the solution to which is

$$
\mathbf{X}_{j}=\mathbf{b} z_{1}^{j}+\mathbf{c} z_{2}^{j},
$$

where $z_{i}$ 's satisfy

$$
E=-(u+v)+v z_{i}+u z_{i}^{-1} .
$$

The second and third equation of (75), can be witten as

$$
\left(\begin{array}{cc}
-(u+\Lambda) Z+\sqrt{u v} & -(u+\Lambda) Z^{-1}+\sqrt{u v} \\
-(v+\Gamma) Z^{L}+\sqrt{u v} Z^{L+1} & -(v+\Gamma) Z^{-L}+\sqrt{u v} Z^{-L-1}
\end{array}\right)\left(\begin{array}{l}
\mathbf{b} \\
\mathbf{c}
\end{array}\right)=0,
$$


where the variable

$$
Z_{i}:=z_{i} \sqrt{\frac{v}{u}}
$$

has been used, in terms of which

$$
E=-(u+v)+\sqrt{u v}\left(Z_{i}+Z_{i}^{-1}\right) .
$$

(77) has nontrivial solutions for $\mathbf{b}$ and $\mathbf{c}$, iff the determinant of the matrix of the coefficients vanishes:

$$
\operatorname{det}\left(\begin{array}{cc}
-(u+\Lambda) Z+\sqrt{u v} & -(u+\Lambda) Z^{-1}+\sqrt{u v} \\
-(v+\Gamma) Z^{L}+\sqrt{u v} Z^{L+1} & -(v+\Gamma) Z^{-L}+\sqrt{u v} Z^{-L-1}
\end{array}\right)=0 .
$$

Again, if in the thermodynamic limit $L \rightarrow \infty$, for all of the solutions of the above equation $\operatorname{Re}\left(\mathrm{Z}+\mathrm{Z}^{-1}\right) \leq 2$, the relaxation time is independent of the reaction rates at boundaries, and the system is in the fast phase. In order that the system be in the slow phase, it is necessary (but not sufficient) that at least one of the roots of (80) has modulus larger than one. For this solution, (80) is simplified in the thermodynamic limit to

$$
\operatorname{det}[\sqrt{u v} Z-(v+\Gamma)] \operatorname{det}[\sqrt{u v} Z-(u+\Lambda)]=0
$$

So, denoting the eigenvalues of $\Lambda$ and $\Gamma$ with $\lambda$ and $\gamma$ respectively, This root must be

$$
Z=\frac{v+\gamma}{\sqrt{u v}}, \quad \text { or } \quad Z=\frac{u+\lambda}{\sqrt{u v}} .
$$

The real parts of the eigenvalues $\lambda$ and $\gamma$ are nonpositive. It is then seen that if $u \geq v$, then the real part of $Z$ in the first case is not greater than 1 , and $\operatorname{Re}\left(\mathrm{Z}+\mathrm{Z}^{-1}\right) \leq 2$. So in this case, the only relevant equation for finding the system in the slow phase is the $Z=(u+\lambda) / \sqrt{u v}$. A similar argument shows that if $v \geq u$, then the first equation of (82) is relevant. So, without loss of generality, let's take $u<v$. In this case, $\mathbf{b}$ should be an eigenvector of $\Gamma$. However, one also demands

$$
\mathbf{s} \mathbf{b}=0 .
$$

If $\mathbf{b}$ is the only eigenvector of $\Gamma$, with zero eigenvalue, then (83) cannot be satisfied, as s would also be the only left eigenvector of $\Gamma$ with zero eigenvalue. So, from the eigenvalues of $\Gamma$, one should put aside one zero eigenvalue, and consider only the other ones. Of course if the zero eigenvalue is degenerate, then $\gamma$ can still be zero.

The system undergoes a dynamical phase transition, when $x$ and $y$ (the real and imaginary parts of $Z$ ) pass the curve

$$
y= \pm(x-1) \sqrt{\frac{x}{2-x}}, \quad x \geq 1 .
$$

In terms of the eigenvalues of $\Gamma$, it is seen that the system is in the slow phase iff

$$
\begin{aligned}
& |\operatorname{Im}(\lambda)|<[\operatorname{Re}(\lambda)+u-\sqrt{u v}] \sqrt{\frac{\operatorname{Re}(\lambda)+u}{2 \sqrt{u v}-\operatorname{Re}(\lambda)-u}}, \\
& \text { or } \operatorname{Re}(\lambda)>2 \sqrt{u v}-u .
\end{aligned}
$$

A simple way to induce the phase transition is to multiply the matrix $\Gamma$ by a parameter $r$. This means multiplying the rates of the reaction at the first site by $r$. As $\operatorname{Re}(\gamma) \leq 0$, one can see that for a large enough value of $r$, the value of $\operatorname{Re}(\gamma)+u-\sqrt{u v}$ will be negative (provided $\operatorname{Re}(\gamma) \neq 0$, that is, provided the zero eigenvalue of the matrix $\Gamma$ is not degenerate). So the system will be in the fast phase. It is also seen that as $r$ tends to zero, either $2 \sqrt{u v}-\operatorname{Re}(\gamma)-u$ becomes negative, or in the first inequality in (86) the right-hand becomes greater than the left-hand side (which tends to zero). So, the system will be in the slow phase. Roughly speaking, increasing the reaction rates at the boundaries, brings the system from the slow phase (relaxation time depending on the reaction rates at boundaries) to the fast phase (relaxation time independent of the reaction rates at boundaries). A similar argument holds, of course, for the case $u>v$ and the eigenvalues of the matrix $\Lambda$.

The details of the calculations can be found in [31].

\section{References}

[1] G. M. Schütz; Exactly solvable models for many-body systems far from equilibrium in Phase transitions and critical phenomena, Vol. 19, edited by C. Domb and J. Lebowitz, (Academic Press, London, 2000).

[2] F. C. Alcaraz, M. Droz, M. Henkel, and V. Rittenberg; Ann. Phys. 230, 250 (1994).

[3] K. Krebs, M. P. Pfannmuller, B. Wehefritz, and H. Hinrichsen; J. Stat. Phys. 78[FS], 1429 (1995).

[4] H. Simon; J. Phys. A28, 6585 (1995).

[5] V. Privman, A. M. R. Cadilhe, and M. L. Glasser; J. Stat. Phys. 81, 881 (1995).

[6] M. Henkel, E. Orlandini, and G. M. Schütz; J. Phys. A28, 6335 (1995).

[7] M. Henkel, E. Orlandini, and J. Santos; Ann. of Phys. 259, 163 (1997).

[8] A. A. Lushnikov; Sov. Phys. JETP 64, 811 (1986); [Zh. Eksp. Teor. Fiz. 91, 1376 (1986)].

[9] M. Alimohammadi, V. Karimipour, and M. Khorrami; Phys. Rev. E57, 6370 (1998).

[10] F. Roshani and M. Khorrami; Phys. Rev. E60, 3393 (1999).

[11] F. Roshani and M. Khorrami; J. Math. Phys. 43, 2627 (2002). 
[12] M. Alimohammadi, V. Karimipour, and M. Khorrami; J. Stat. Phys. 97, 373 (1999).

[13] A. Aghamohammadi and M. Khorrami; J. Phys. A 33, 7843 (2000).

[14] M. Alimohammadi, and N. Ahmadi; Phys. Rev. E 62, 1674 (2000).

[15] F. Roshani and M. Khorrami; Phys. Rev. E 64, 011101 (2001).

[16] M. A. Burschka, C. R. Doering, and D. ben-Avraham; Phys. Rev. Lett. 63, 700 (1989).

[17] D. ben-Avraham; Mod. Phys. Lett. B 9, 895 (1995).

[18] D. ben-Avraham; in Nonequilibrium Statistical Mechanics in One Dimension, edited by V. Privman, pp 29-50 (Cambridge University press, 1997).

[19] D. ben-Avraham; Phys. Rev. Lett. 81, 4756 (1998).

[20] T. Masser, D. ben-Avraham; Phys. Lett. A 275, 382 (2000).

[21] M. Henkel and H. Hinrichsen; J. Phys. A34, 1561 (2001).

[22] M. Alimohammadi, M. Khorrami, and A. Aghamohammadi; Phys. Rev. E 64, 056116 (2001).

[23] M. Khorrami, A. Aghamohammadi, and M. Alimohammadi; J. Phys. A 36, 345 (2003).
[24] M. Mobilia and P. A. Bares; Phys. Rev. E 64, 066123 (2001).

[25] A. Aghamohammadi, M. Alimohammadi, and M. Khorrami; Eur. Phys. J. B 31, 371 (2003).

[26] G. M. Schütz; J. Stat. Phys. 79, 243 (1995).

[27] A. Aghamohammadi, A. H. Fatollahi, M. Khorrami, and A. Shariati; Phys. Rev. E 62, 4642 (2000).

[28] A. Shariati, A. Aghamohammadi, and M. Khorrami; Phys. Rev. E 64, 066102 (2001).

[29] M. Khorrami and A. Aghamohammadi; Phys. Rev. E 63, 042102 (2001).

[30] A. Aghamohammadi and M. Khorrami; J. Phys. A 34, 7431 (2001).

[31] N. Majd, A. Aghamohammadi, and M. Khorrami; Phys. Rev. E 64, 046105 (2001).

[32] M. Khorrami and A. Aghamohammadi; Phys. Rev. E 65, 056129 (2002).

[33] R. J. Glauber; J. Math. Phys. 4, 294 (1963).

[34] G. M. Schütz; Eur. Phys. J. B 5, 589 (1998). 